\title{
Active ABF for Sonar
}

\author{
James M. Alsup \\ Naval Command, Control and Ocean Surveillance Center \\ Research, Development, Test and Evaluation Division \\ San Diego, California 92152
}

\begin{abstract}
An adaptive beamforming (ABF) algorithm tailored for active sonar is described. It is especially suited for processing $F M$ waveforms received by a fixed or moving sensor array. Adaptation is confined to small windows of time whose duration is closely related to the rangeresolution of the FM ping. This leads to the use of a "time series" of covariance matrices formed from the matched-filter response of the sensor outputs.
\end{abstract}

\section{Introduction}

This paper describes an approach to $A B F$ which adapts each delay bin rather than each frequency bin, resulting in a method which is directly matched to the active scenario. This technique has been shown to work for frequency-modulated waveforms commonly used in active sonar: linear frequency modulation (LFM) and hyperbolic frequency modulation (HFM). It is applicable to any three-dimensional sensor configuration, within the limits of uniformity of the ocean medium. Certain simplifications will result when a uniformly-spaced line array is used.

The algorithm flow is diagramed in Figure 1, and is presented in five sections: data acquisition; matched filter; covariance analysis; EV/EV analysis; and beamforming. Experimental results are shown using data recorded from a horizontal planar array during a Navy test.

\section{Data acquisition}

The method described herein can be used with a large variety of array types, including horizontal line arrays, horizontal planar arrays, and arrays which are sparse or which are filled. These arrays are primarily characterized by the number of hydrophone elements, $N$, which partially determines or limits the effectiveness of the adaptation in the presence of multiple targets and/or reverberation.

One can thus acquire a matrix of digital data, where each row contains the time-series output for one of the array sensors, and each column is the ordered output of all array sensors for a given sample instant. The length of the time series (row) is chosen to encompass the return from a single ping.

\section{Matched filter}

The digital replica of the transmitted waveform, modified by the application of a window function (e.g., Hamming), is used to compute the aperiodic cross-correlation between the replica and each row of the input data matrix, resulting in a matrix of matched-filtered (MF) sensor data. This matrix is checked for data quality; in

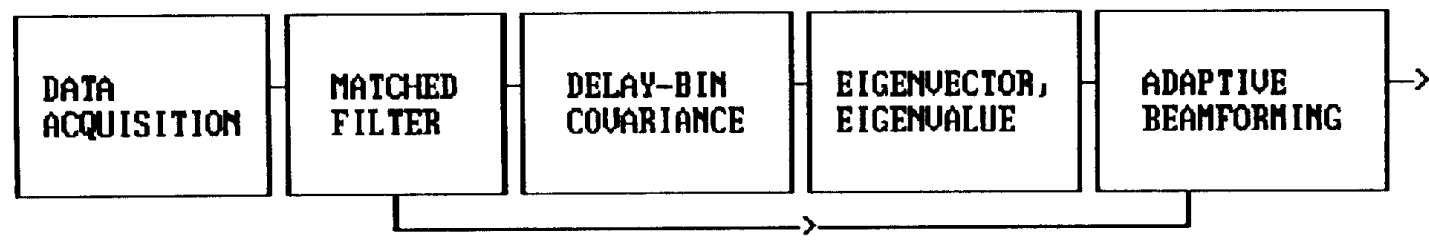

Figure 1. ABF Signal Processing Flow Diagram 
the case of bistatic sonar (transmitter and receiver not co-located), normalization based on the magnitude of the direct blast (DB) can be performed.

The bandwidth of the transmitted waveform can be selected to be sufficiently large to provide adequate resolution of the potential sonar targets, yet sufficiently small to allow sensor responses from opposite sides of the array to overlap (and thus form cross-terms in the covariance matrix).

We note that each sensor's DB MF response (or any MF peak) is comprised of an envelope and a singlefrequency carrier. In the case of an LFM signal, a derivation of this fact is presented in reference [1]. A similar proof can be given for an HFM signal. Thus, beamforming can be performed "at a single frequency" even though the individual target and reverberation features are characterized by the bandwidth of the transmitted ping.

\section{Covariance and EV/EV analysis}

Next, a covariance matrix is estimated for each delayresolution cell associated with the sensor MF outputs. Typically, delay resolution cells are overlapped by 50 $75 \%$ so as to capture a maximum response for a given target echo or reverberation feature. The result is a collection of covariance matrices representing a history of response over the duration of the ping interval. Each covariance matrix is of size $\mathrm{NxN}$, and is associated with the center frequency of the transmitted FM waveform. One can associate a given estimated covariance matrix with the echoes and reverberation emanating from a narrow elliptical annulus whose foci are the positions of the source and receiver.

Then, eigenvector/eigenvalue (EV/EV) analysis is performed on each estimated covariance matrix, resulting in a collection of $\mathrm{NxN}$ eigenvector matrices, one for each delay resolution cell. It is necessary that the number of sensors $\mathbf{N}$ be chosen to be larger than the expected number of targets and reverberators contained in any one of the elliptical annuli described above.

\section{Beamforming}

Beamforming is performed by selecting an eigenvector matrix (with associated eigenvalues) to determine the weights in a single-frequency MVDR algorithm (or other selected high-resolution method) for a particular delay bin, and beams are formed over a selected range of azimuth [6]. A reasonable set of elevation beams are also computed, and the maximum elevation response is selected for each of the computed azimuth beams. This is repeated for other bins in the delay-range of interest. Finally, a threshold is determined using median and/or average levels seen in the selected delay/azimuth sector, and a display of this sector is presented to an operator for evaluation.

In the present work, three beamformer types have been used: MVDR [2-3], MUSIC [4-5], and conventional (CBF). Once an echo is identified, its delay and azimuth parameters can be used to determine the location of the target or reverberator relative to the source and array

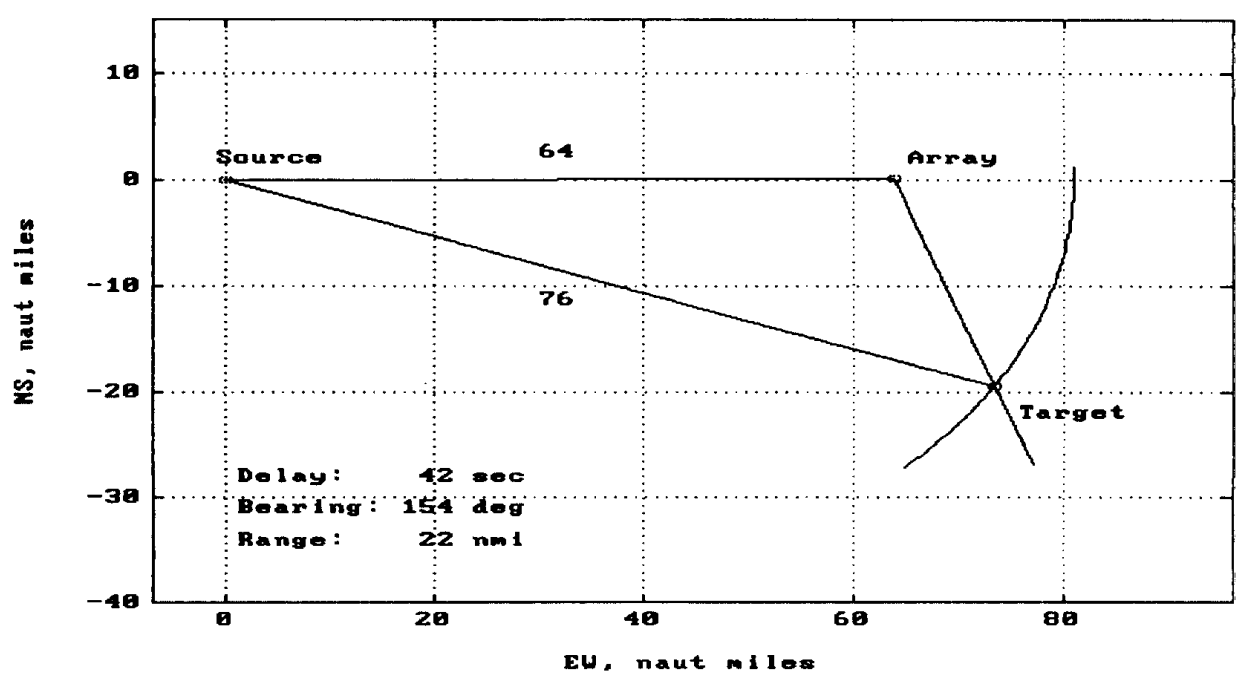

Figure 2. Active bistatic configuration, 1991 

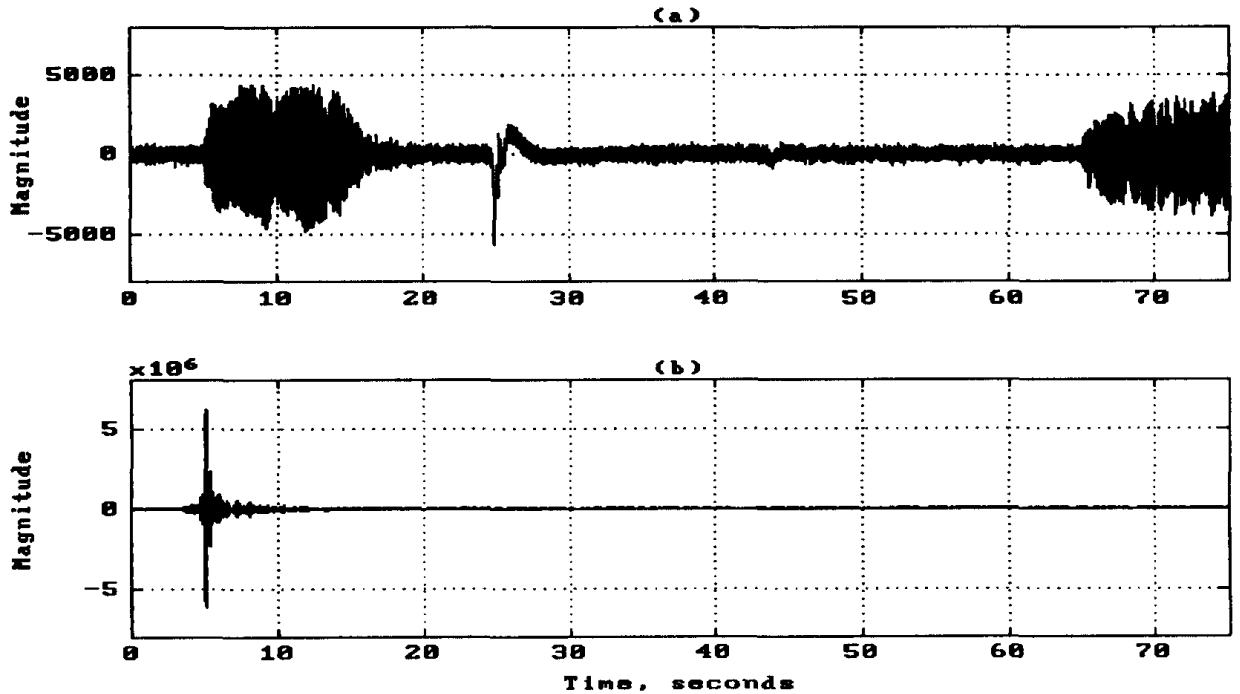

Figure 3. Single sensor data, 1991: a) direct; b/ via matched filter

positions, using the intersection of the associated line of bearing with the elliptical line of position. This same echo can be further evaluated using auxiliary plots of the $\mathrm{ABF}$ output versus delay (at optimum az/el), versus azimuth (at optimum del/el), versus elevation (at optimum del/az), or any pair of these variables (at optimum of the third).

\section{Results with real data}

Data was collected during a Navy test in 1991, involv- ing a fixed acoustic source, a cooperative underwater target, and a number of different sonar receive arrays. The active bistatic configuration for the array considered here (a sparse, horizontal planar array) consisted of a source located toward the West, with array and target toward the East (see Figure 2). Approximate distances among these were 64 miles (source-to-array), 76 miles (source-to-target), and 22 miles (array-to-target).

A large number of HFM pings with different center frequencies and various bandwidths were transmitted from the source. Figure 3a shows a typical 75-second

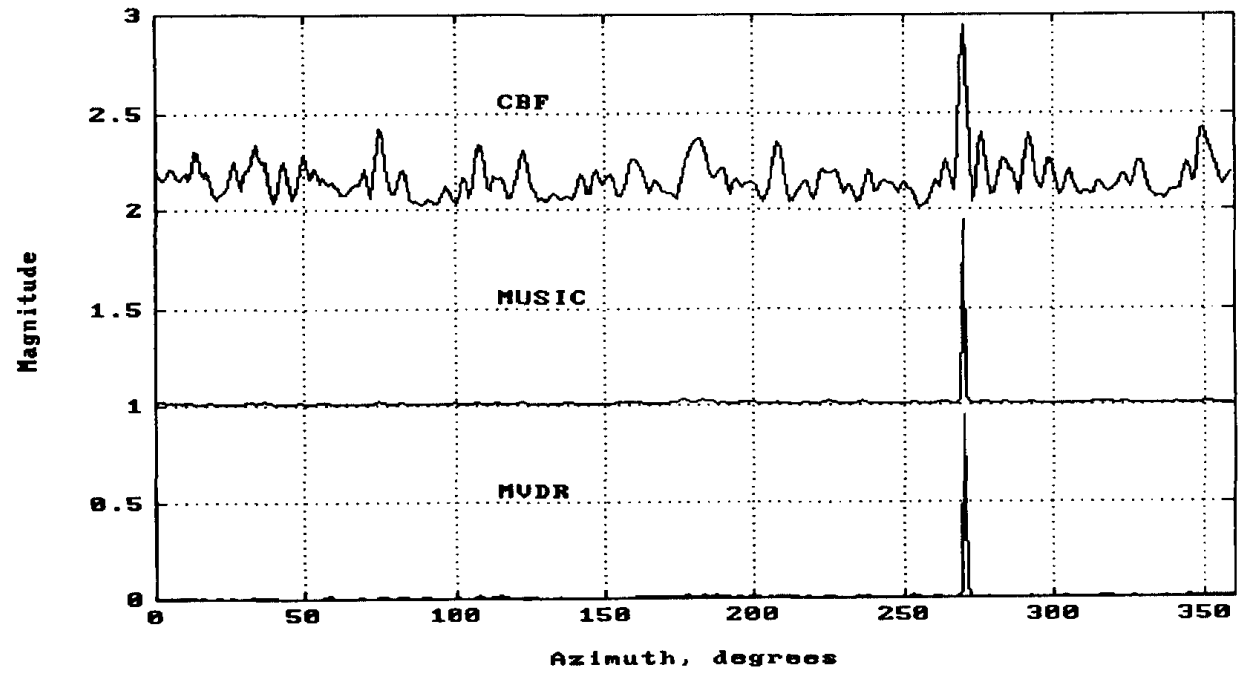

Figure 4. DB beamformer outputs versus azimuth 


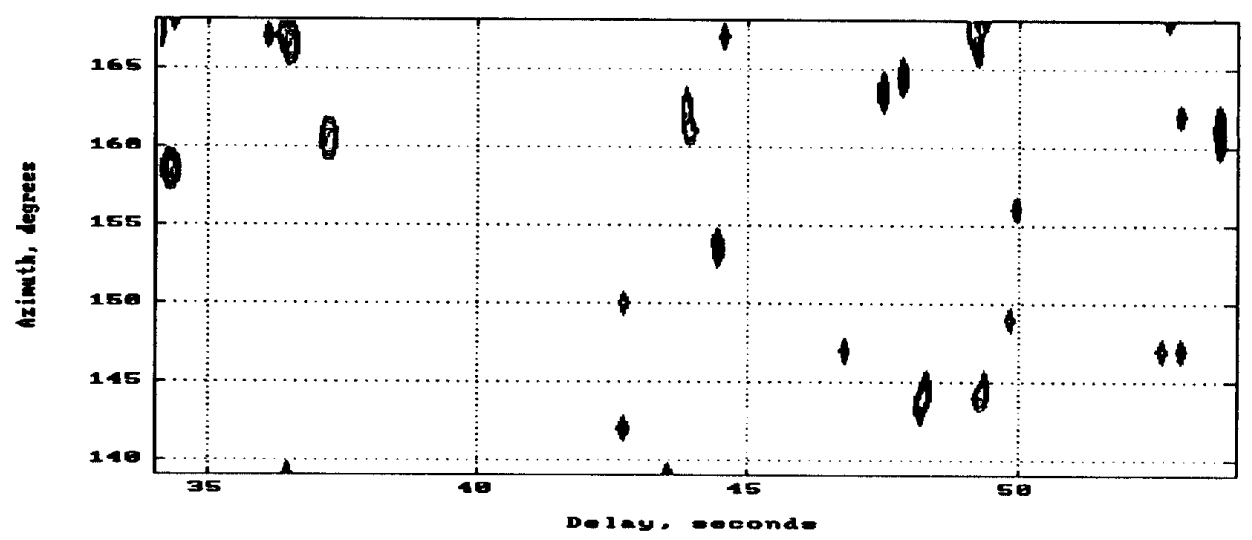

Figure 5. MVDR echo, beamformer contour versus delay/azimuth

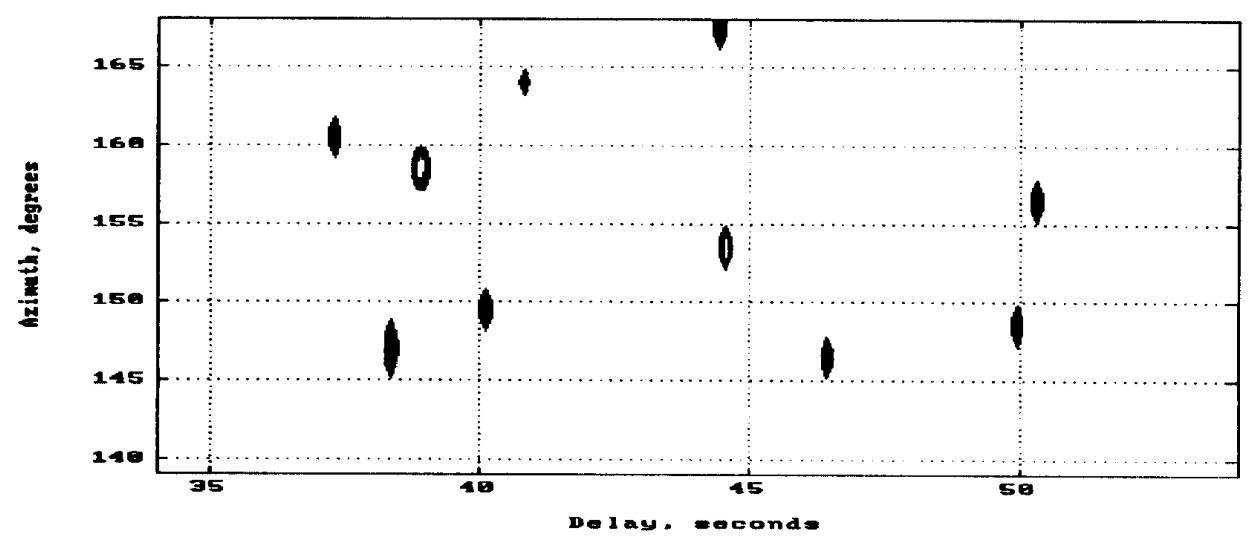

Figure 6. MUSIC echo, beamformer contour versus delay/azimuth

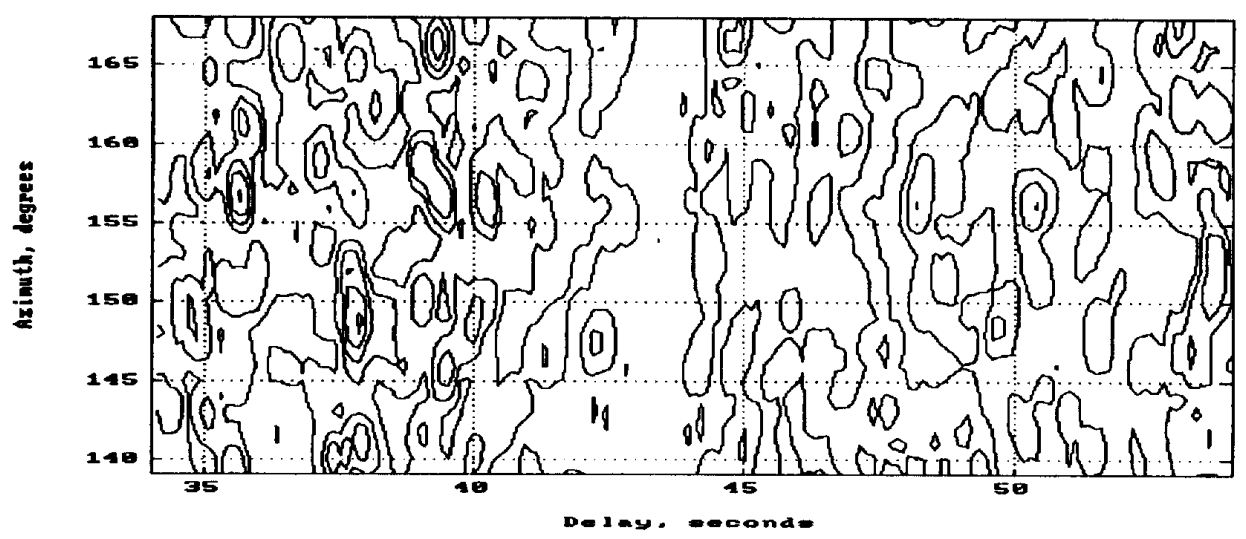

Figure 7. CBF echo, beamformer contour versus delay/azimuth 
record for a single sensor, in which two successive pings with different center frequency can be seen. The DB can be seen on a single sensor, but echoes are not seen. The MF output of the 75-second record for this sensor, using a replica for the first ping, is shown in Figure $3 \mathrm{~b}$.

Figure 4 shows three different beamformer outputs of the direct blast signal versus azimuth, computed at the optimum delay and elevation of the DB. As can be seen, the CBF beamformer has much higher sidelobe levels than either the MVDR or MUSIC beamformers, and thus its performance is much worse than theirs.

Figures 5, $6 \& 7$ show three echo output presentations for the subject ping. These presentations are twodimensional plots of beamformer output magnitude within a sector of delay/azimuth space. The chosen sector is centered on the known position of the underwater target, and is 20 seconds by 30 degrees. The actual target location (from ground truth) is centered in the sector, where a likely response is seen for the MVDR (Figure 5) and MUSIC (Figure 6) beamformers. The estimated delay between DB and echo is computed to be about 39 seconds (or 44 seconds from the beginning of the ping interval). The CBF output (Figure 7) at these coordinates is too small to be detected, being occluded by a plethora of sidelobes.

Echoes and reverberation originating from the same elliptical annulus may constitute coherent target returns, and thus be unresolvable using the ABF described above. This is similar to the problem of resolving coherent multipath in the same frequency bin in the case of passive sonar.

Some of the receivers used at this Navy test are uniformly-spaced line arrays, in which case the method of subarray smoothing [7-8] can be used to make the algorithm work in the presence of coherent echoes. Some success has been demonstrated on Navy data using these techniques [9-10]. For planar arrays or non-uniformly spaced line arrays, other smoothing methods may be used.

\section{Summary}

The $\mathrm{ABF}$ algorithm described herein uses a new way to form the estimated covariance matrix based on information contained in a delay bin rather than in a frequency bin. This results in a method which is sensitive to the transient features of the matched-filtered beam outputs, yet contains enough information to be effective in spatial discrimination of these features. This algorithm has been found to provide a significant improvement in performance relative to the CBF algorithm and modified versions of passive-ABF algorithms used heretofore.

\section{Acknowledgements}

This work was supported by the Space and Naval Warfare Systems Command, PMW-183.

\section{References}

1. C.E. Cook \& M. Bernfeld, Radar signals, an introduction to theory and application, p. 133, Academic Press, New York, 1967.

2. J.W.R. Griffiths, "Adaptive array processing, a tutorial," IEE Proc, 130:F;H, pp. 3-10, February 1983.

3. H. Cox, R.M. Zeskind, and M.M. Owen, "Robust adaptive beamforming," IEEE Trans. ASSP, 35, pp. 1365-1376, October 1987

4. R.O. Schmidt, "Multiple emitter location and signal parameter estimation," in Proc. RADC Spectral Estimation Wbrkshop, Rome, NY, pp. 243-256, 1979.

5. R.O. Schmidt, "A signal subspace approach to multiple emitter location and spectral estimation," Ph.D. dissertation, Stanford Univ., 1981.

6. J.M. Alsup, "Adaptive beamforming for active sonar," Advanced Signal Processing Algorithms, Architectures, and Implementations IV, SPIE Vol. 2027, Paper 47, July 1993.

7. T.J. Shan, M. Wax, and T. Kailath, "On spatial smoothing for direction-of-arrival estimation of coherent signals," IEEE Trans. ASSP, 33, p. 806-811, Aug. 1985.

8. B.D. Rao and K.V.S. Hari, "Weighted subspace methods and spatial smoothing: analysis and comparison, IEEE Trans. Signal Processing, 41, pp. 788-803, February 1993.

9. J.C. Lockwood, "Broadband adaptive beamformer for linear-frequency-modulation active signals," Proc. NATO Ocean Reverberation Symposium, La Spezia, Italy, May 1992.

10. J.C. Lockwood, "Active LFM mixer adaptive (ALMA) beamformer," presented at 4th Navy R\&D Information Exchange Conference, San Diego, California, April 1993. 